\title{
Small Colony Variants of Staphylococcus aureus: Missed Entities?
}

\section{IJCRR \\ Section: Healthcare \\ Sci. Journal Impact \\ Factor: 6.1 (2018) \\ ICV: 90.90 (2018)}

\section{Sathyanarayan Muthur Shankar, Kusuma Gowdra Rangappa, Ambica Rangaiah, Shantala Gowdara Basawarajappa*}

Department of Microbiology, Bangalore Medical College and Research Institute, K.R. Road, Fort, Bengaluru - 56ooo2, lndia.

\section{Copyright@IJCRR}

\section{ABSTRACT}

Staphylococcus aureus is among the predominant pathogens in clinical practice. Small colony variants(SCV) of staphylococci are regarded as one of the mechanisms for resisting therapy that extends beyond the classic forms of resistance. They are slowgrowing subpopulations of Staphylococcus aureus (S.aureus) which are often associated with persistent or recurrent infections and frequently missed for diagnosis. The present study was undertaken to determine the prevalence of SCV of S.aureus among clinical specimens received from in-patients and out-patients attending attached hospitals of a tertiary care centre.

Methodology: The present study was undertaken as a laboratory-based descriptive study, carried out in the Department of Microbiology in a tertiary care hospital over two years from May 2018 to April 2020. After obtaining ethicalclearance for the study, a total of 1000 consecutive S.aureus isolates from different clinical samples routinely received in the laboratory from outpatient and inpatient departments were screened for SCVs. The clinical specimens were processed as per standard microbiological protocols. Phenotypic characters were noted along with auxotrophy and antimicrobial susceptibility. The isolates were also characterized by molecular methods for nuc and mec $A$ genes.

Results: 6 isolates of SCV were noted during the study among clinical specimens, 4being HA-MRSA and 2 CA-MRSA. All these isolates were found to be methicillin-resistant. The SCVs exhibited delayed coagulase and heminauxtotrophy. nuc and mec $A$ genes were detected by a polymerase chain reaction and confirmed as methicillin-resistant S.aureus.

Conclusion: In the present study, 6 isolates of SCV were noted. Detection of SCV poses a challenge in laboratory practice. The high degree of suspicion and meticulous reporting of SCV aid inappropriate management of cases.

Key Words: Small colony variants, S.aureus, HA-MRSA, CA-MRSA, nuc gene

\section{INTRODUCTION}

Staphylococcus aureus, apart from being a common human commensal, is a remarkably versatile pathogen that can cause a broad range of human infections. Coupled with an extraordinary capacity to develop drug resistance and the emergence of community-circulating highly virulent strains, S.aureusis a major threat to human health in both the hospital and the community. Past studies have shown that staphylococci have mechanisms for resisting therapy that extends beyond the classic forms of resistance. The formation of slowgrowing subpopulations of cells designated "small colony variants" (SCVs)may represent one such mechanism. ${ }^{1}$

SCVs are naturally occurring subpopulations of Staphylococcus aureus and are associated with persistent or recurrent infection. They exhibit small colony sizes due to their slow rate of growth and are also characterized by decreased pigmentation and hemolysis. The variants may be identified by their atypical colonial morphology with tiny, mostly non-pigmented and nonhemolytic colonies slowly growing following 24-72 hours incubation on rabbit blood agar. Unusual biochemical reactions-mannitol salt agar negative and reduced coagulase activity (incubation for $>18$ hours is needed) are also typical features of the variants as compared to the normal phenotype of S.aureusisolates. Due to these characteristics, isolates suspicious for S.aureusSCVs should be confirmed as S.aureusmolecularly by testing the speciesspecific nuc gene and coa genes. ${ }^{2}$

SCVs gain a survival advantage from their ability to hide within host cells, which protects this naturally occurring subpopulation of S.aureusfrom host defences and decreases exposure to antibiotics. The generation time for S.aureusSCVs

\section{Corresponding Author:}

Shantala Gowdara Basawarajappa, Department of Microbiology, Bangalore Medical College and Research Institute, Victoria Hospital Campus, K.R. Road, Fort, Bengaluru - 560002. India; Ph: +91-9448078081; E-mail: drshantalagb@gmail.com

ISSN: 2231-2196 (Print)

Received: 02.06 .2020 ISSN: $0975-5241$ (Online)

Revised: 28.06 .2020
Published: 08.08 .2020 
is six- to nine-fold longer than that for metabolically normal S.aureus, resulting in tiny colonies that are frequently not visible before 48 to 72 hours of incubation. Consequently, correct identification and susceptibility testing for clinical laboratories become complicated, which may result in diagnostic underestimation and therapeutic failures. The combination of SCV phenotype and methicillin resistance poses a major challenge to diagnosis and therapy. SCVs of S.aureusrepresent under-diagnosed agents of persistent subclinical infections that need to be promptly diagnosed and treated. ${ }^{3,4}$

SCVs have been described in cases of sepsis, cystic fibrosis, soft-tissue infection, osteomyelitis, arthritis, brain abscess, sinusitis, and in some foreign body-associated infections. These variants are difficult to treat because of their increased resistance to aminoglycosides and cell-wall-active antibiotics, as well as their ability to persist. ${ }^{5}$

SCVs of S.aureusoften pose a diagnostic dilemma for laboratory personnel and treating physicians. The present study was undertaken to characterize SCV by both phenotypic and genotypic methods as well as to evaluate different methods to detect methicillin resistance in SCV from a tertiary care hospital.

\section{Aims and Objectives:}

1. To isolate and characterize the SCVs of Staphylococcus aureus by phenotypic and genotypic methods

2. To study the prevalence of SCVs in Community acquired (CA) strains of Staphylococcus aureus

3. To evaluate different methods of detection of methicillin resistance in SCV's including mec $A$ gene by PCR

\section{MATERIALS AND METHODS}

The present study was undertaken as a laboratory-based descriptive study, carried out in the Department of Microbiology in tertiary care hospital over 2 years from May 2018 to April 2020. Institutional Ethics Committee (IEC) approval was sought before the commencement of the project. 1000 consecutive S.aureus isolates from various clinical samples routinely received in the laboratory from outpatient and inpatient departments were screened for SCVs; Community acquired (CA) and Hospital-acquired (HA) SCV strains to be in the ratio of 1:1 (Figure 1).

Clinical specimens were cultured on 5\% sheep blood agar (BA), MacConkey agar (MA), Mannitol Salt Agar (MSA) and Brain-Heart Infusion Agar (BHIA), with $5 \% \mathrm{NaCl}$ (BHIA $5 \%-\mathrm{NaCl}$ ). All agar plates were incubated at $37^{\circ} \mathrm{C}$ for at least 48 hours aerobically, h for BHIA $5 \% \mathrm{NaCl}$ plates, which were incubated anaerobically.

Non-haemolytic, non-pigmented, pinpoint or fried-egg colonies on BA and small colonies on MSA and BHIA $5 \% \mathrm{NaCl}$, presumptively identified as SCVs were then inoculated on Columbia blood agar (CBA) and Schaedler agar (SA). Inoculated plates of CBA were incubated in a normal atmosphere and SA were incubated in $5-10 \% \mathrm{CO}_{2}$, both at $35^{\circ} \mathrm{C}$. If the colonies were noted to be of normal size, haemolytic and pigmented on SA, they were considered to as SCVs (Figure 2). These strains were further subjected to species identification by Gram staining, catalase reaction and tube coagulase result. Identification of S.aureus was confirmed by nuс PCR.

Auxotrophy for hemin, thymidine and menadione were tested with standard disks impregnated with haemin $(5.4 \mu \mathrm{g})$, thymidine $(1.5 \mu \mathrm{g})$ and menadione $(1.5 \mu \mathrm{g}$, Sigma), respectively. A strain was considered positive for auxotrophy if a zone of growth surrounding the impregnated disks on Mueller-Hinton agar was noted after 24 hours of incubation at $35^{\circ} \mathrm{C}$ (Figure 3).

The following susceptibility testing methods were evaluated for their abilities to detect methicillin resistance in SCVs in comparison with normal-phenotype strains: disc diffusion test on Mueller-Hinton agar, supplemented with $2 \% \mathrm{NaCl}$ $\left(30^{\circ} \mathrm{C}\right)$ with $30 \mu \mathrm{g}$ cefoxitin discs; MRSA-Screen latex agglutination test (Denka Seiken Co. Ltd., Tokyo, Japan) (Figure 4). Determination of the presence of the mec $A$ gene was performed by PCR. ${ }^{3,6,7}$ The prevalence of SCV in CA and HA strains of S.aureus were determined.

Antimicrobial susceptibilities of the strains were determined by Vitek-2 (Biomerieux) and Kirby Bauer disc diffusion method using gentamicin, ciprofloxacin, linezolid, trimethoprim-sulphamethoxazole following Clinical Laboratory Standards Institute guidelines, 2018. Strains with a normal phenotype were tested on Mueller-Hinton agar, and SCVs were tested on brain-heart infusion agar. Methicillin sensitive S.aureus (MSSA) and Methicillin-resistant S.aureus (MRSA) were noted. ${ }^{8}$

Antibiotic susceptibility to oxacillin and cefoxitin among clinical isolates of S.aureuswas carried out using Vitek-2 GPCID AST628 susceptibility card (Biomerieux). Suspensions of cultures were made and loaded into the test cards according to the manufacturer's instructions. Cefoxitin screen positive isolates; Oxacillin resistant isolates with MIC $\geq 4$ $\mu \mathrm{g} / \mathrm{ml}$ were considered as MRSA.

Conventional PCR was performed on the SCV isolates to detect nuc for confirmation of S.aureus and mecA gene for the mechanism of methicillin resistance.

\section{Detection of mec A and nuc genes using con- ventional PCR}

To detect mecA and nuc genes, bacterial culture were lysed, DNA extracted by boiling method, and gene-specific primers were used to a polymerase chain reaction (PCR) amplifying DNA fragments. 
The primer sequences and product length for mec $A$ and nuc used for the study were:

mecA- Forward 5'-TCCAGATTACAACTTCACCAGG-3' and

\section{Reverse- 5'-CCACTTCATATCTTGTAACG-3' [162 bp];} nuc-Forward 5'- GCGATTGATGGTGATACGGTT-3' and

Reverse-5 '-AGCCAAGCCTTGACGAACTAAAGC-3' [270bp];

The amplification products were analyzed using $2 \%$ agarose gel electrophoresis with ethidium bromide dye under UV transilluminator. ${ }^{9}$

The data were entered in Excel for analysis. The data were analysed using SPSS software version 20.0. The data has been expressed in proportion and association between categorical data was analysed using appropriate statistical methods. The diagnostic efficacy was addressed using sensitivity, specificity and diagnostic efficacy test.

\section{RESULTS}

In the present study, a total of 1000 S.aureus isolates from patients attending the attached hospitals of BMCRI, Bengaluru were included, from in-patients and out-patients (Figure 1). The sex distribution of cases included in the study shows a male predominance among both hospitalized and out-patients (Figure 5). Among the isolates recovered from CA infections, MSSA was predominant and noted in 337 isolates (67.4\%) whereas MRSA was predominant in HA infections, being noted in 352 isolates (70.4\%) (Table 1). Among SCV isolates, $100 \%$ methicillin resistance was detected. 6 isolates of S.aureus were determined to be small colony variants. Of these, 4 isolates were from hospitalized patients and 2 from outpatients.

$6(0.6 \%)$ isolates were found to be SCV in the present study. These isolates were from patients with an infected orthopaedic implant (1), osteomyelitis (2), Otitis media (1), Lower Segment CaesarianSection(LSCS) wound (1) and sputum (1). 5 of these 6 patients were males $(83.33 \%)$.

All SCV isolates were positive for catalase and coagulase (delayed, 18 hours) and were mannitol non-fermenters. All isolates of SCV were found to be methicillin-resistant, indicating the presence of both HA-MRSA and CA-MRSA (Figure 6). The results of the Kirby-Bauer method were $100 \%$ concordant with the results of Vitek-2 and latex agglutination tests. The SCV isolates showed reduced susceptibility to gentamicin, ciprofloxacin and trimethoprimsulphamethoxazole, but all isolates were susceptible to linezolid (Table 2).
$2 \mathrm{SCV}$ isolates encountered in the present study exhibited auxotrophic for hemin (Figure 3). However, none of the isolates showed auxotrophic for thymidine and menadione. All $\mathrm{SCV}$ isolates in the study, when further subcultured on BA were noted to gain classical morphology of S.aureus, thereby indicating unstable mutation.

All SCV isolates detected in the study were positive for both nuc and mecA genes, confirming the identification as S.aureus and mechanism of resistance respectively (Figures 7 and 8 ). The sensitivity, specificity and diagnostic efficacy of the tests performed for detection of methicillin resistance were found to be $100 \%$.

\section{DISCUSSION}

Small colony variants of S.aureuswere described almost 100 years ago. These have been described as "dissociates" or "dwarf" and "G" ("gonidial") colonies in various studies. Clinical reports and distinct SCV-associated syndrome have been described. However, there is a lack of controlled trials of therapy in these cases.

SCV may exhibit loss of $\alpha$-hemolysin activity, apart from auxotrophic for thymidine and menadione or hemin. The $\mathrm{SCV}$ isolates encountered during the present study exhibited heminauxotrophy.SCVs originate from wild-type strains by a genetic mutation. SCVs exhibiting auxotrophism is reported to have deficiencies in the electron transport and the thymidylate biosynthetic pathway. SCVsnoted in the present study, when further subcultured on blood agar were noted to gain classical morphology of S.aureus, thereby indicating that these may represent unstable mutants. Intra-cellular persistence shields them from host immunity and antimicrobials. These are associated with persistent, relapsing and chronic infections like cystic fibrosis, osteomyelitis, chronic wound infections, implant-related, device-associated infections. SCVs characteristically exhibit slow growth rate, absence of pigment, tiny colonies and atypical biochemical characteristics, making laboratory diagnosis very difficult. SCV are resistant to aminoglycosides and trimethoprim-sulphamethoxazole. Effectiveness of cell-wall active antimicrobials is also reduced. ${ }^{10,11,12}$

In the present study, all SCV isolates were noted to be susceptible to Linezolid. However, the effectiveness of antimicrobials like aminoglycosides, fluoroquinolones and trimethoprimsulphamethoxazole were found to be limited. Small colony variants (SCVs) are naturally occurring subpopulations of $S$. Aureus and are associated with persistent or recurrent infection. They differ from normal-phenotype not only in their small colony sizes (because of their slow rate of growth) but also by decreased pigmentation and hemolysis. The variants may be identified by their atypical colonial morphology with 
tiny, mostly non-pigmented and nonhemolytic colonies slowly growing following 24-72 hours incubation on rabbit blood agar. The SCVs may be confusing for laboratory physicians due to variations in their biochemical reactions (MSA negative), and reduced coagulase activity (incubation for over 18 hours required). Isolates suspicious for S.aureusSCVs should be confirmed as S.aureusmolecularly by testing the speciesspecific nuc and coa genes. $^{2}$

All isolates of suspected SCVs in the present study were tested for $n u c$ gene and confirmed as S.aureus. In the present study, we encountered 6 small colony variants in a total of 1000 S.aureusisolated from clinical specimens from CA and HA infections.

S. Yagci et al evaluated a total of 519 respiratory specimens from 248 cystic fibrosis cases for SCVs. A majority of these specimens were sputum $\{209(40.3 \%)\}$, and deep throat swab specimens [310 (59.7\%)] were deep throat swab samples.S. Yagci et al reported a median age to be 9.9 years (range: 1-58 years) and was similar in the two genders. They also reported SCV prevalence of $16.2 \%$ in their study, with a predominance of SCV positivity among females [13(65\%)]. In the present study, all SCV isolates were noted in the age group of 15-45 years and the most isolates of SCV were noted among males. ${ }^{6}$

S.Bhattacharyya et al in their study showed that out of a total of 71 different isolates of $S$. aureus isolated and identified in the laboratory during these 11 months, 11 were found to be small colony variants (15.5\%). Out of these $11 \mathrm{SCV}$ isolates, 5 were recovered from pus samples, 2 each from urine samples and wound discharge, and 1 each from sputum (respiratory tract) and central venous catheter tip. Out of these 11 patients, 9 were female subjects and only 2 were male. Thus, a gross female preponderance was observed. All these isolates were coagulase positive, but 9 out of these 11 isolates $(81.8 \%)$ were delayed coagulase-positive, and that too, after adding excess plasma. All were mannitol non -fermenters and catalase-positive. Only $4 \mathrm{SCV}$ isolates (36.4\%) were auxotrophic for Carbon-dioxide. No Vitamin-K auxotroph was found. Out of the remaining 7, 3 were Thymidine auxotroph and the rest 4 showed indeterminate auxotrophism. Only 2 isolates (18.18\%) were Cefoxitin resistant, correlating with Methicillin resistance. The corresponding figures for Amikacin, Levofloxacin, Erythromycin, Netilmicin, Cotrimoxazole and Clindamycin were $0 \%, 9.09 \%, 27.27 \%$, $9.09 \%, 27.3 \%$ and $18.18 \%$, respectively. In the present study, 5 of the $6 \mathrm{SCV}$ isolates were from males. These isolates exhibited delayed coagulase positivity and were mannitol nonfermenters. $2 \mathrm{SCV}$ isolates showed heminauxotrophy in the present study, with no auxotrophy demonstrated against thymidine and menadione. The antimicrobial susceptibility pattern of these isolates is comparable to the results of the study by S. Bhattacharyya et al. ${ }^{7}$

\section{CONCLUSION}

A total of 6 small colony variants $(0.6 \%)$ of S.aureus were encountered during the study among inpatient and outpatient clinical specimens, predominantly among hospitalized patients (66.66\%) and males (83.33\%). 2 of these SCV isolates exhibited auxotrophism for hemin. All SCV isolates noted during the present study were found to be methicillin-resistant. SCVof S.aureusare associated with persistent infections and are frequently missed for diagnosis in clinical settings and resulting in improper management of cases. Meticulous reporting is the need of the hour for identification of small colony variants of S.aureus and appropriate management of cases.

Abbreviations: SCV: Small colony variants, S.aureus: Staphylococcus aureus, PCR: Polymerase chain reaction, HA: Hospital-acquired, CA: Community-acquired, BA: Sheep blood agar, MA: MacConkey agar, MSA: Mannitol Salt Agar, BHIA: Brain-Heart Infusion Agar, CBA: Columbia blood agar, SA: Schaedler agar, MSSA: Methicillin sensitive S.aureus, MRSA: Methicillin-resistant S.aureus, DNA: Deoxyribonucleic acid, MIC: Minimum inhibitory concentration

Acknowledgements: The authors sincerely acknowledge the technical assistance provided by Dr Renji Francis, Department of Microbiology, BMCRI and Ms Asha Gowda, Research Assistant, State Level Virus Research and Diagnostic Laboratory, BMCRI, Bengaluru

Funding: Advanced Research Wing, Rajiv Gandhi University of Health Sciences, Karnataka Grant-in-Aid for an advanced research project for the year 2016-17 vide reference ORDER NO. RGU: RGU/ADV.RES/GRANTS/059/2016-17 Dated:30.01.2017

\section{Conflict of interest: None}

\section{REFERENCES}

1. Sifri Costi D, Baresch-Bernal A, Stephen B. Calderwood,and Christof von Eiff. Virulence of Staphylococcus aureus Small Colony Variants in the Caenorhabditiselegans. Infection Model Inf. And Imm., 2006, 74(2): 1091-1096

2. Bhattacharyya S, Kumar D.Small colony variants of Staphylococcus aureus: Enemies with hidden weapons .Indian Journal of Medical Microbiology 2014;32(4): 460-461.

3. Parham S, Markus R,Peter G, Reno F,Peter E. Ochsner. Staphylococcus aureusSmall Colony Variants in Prosthetic Joint Infection, Clinical Infectious Disease 2006;43 :961- 967.

4. Frank K, Karsten B, Georg P, and Christof von E. Evaluation of Different Methods To Detect Methicillin Resistance in SmallColony Variants of Staphylococcus aureus Journal Of Clinical Microbiology 2004; 42:1277-1279.

5. Nahed Al Laham. Mini-review: Formation, antibiotic resistance and clinical outcome of infections associated with small colony variants of staphylococci. Science, Technology and Education Formatex 2013 493-50. 
6. Yagci S, Hascelik G, Dogru D, Ozcelik U and Sener B. Prevalence and genetic diversity of Staphylococcus aureus smallcolony variants in cystic fibrosis patients. ClinMicrobiol Infect 2013; 19: 77-84.

7. Bhattacharyya S, Sarfraz B,.Jaiswal N.K. Roy C,H. Kumar D,R. Prasad E,R Shristig F, Ahmed S. Characteristics And Antibiogram Of Small Colony Variants of Staphylococcus AureusFrom A Tertiary Care Hospital In Eastern India. Indian J.Sci.Res 2015; 11 (1): 006-008.

8. CLSI. Performance Standards for Antimicrobial Susceptibility Testing. 28th ed. CLSI supplement M100. Wayne, PA: Clinical and Laboratory Standards Institute; 2018

9. Cho, Joon-Il, Hye-Jin Jung, Young-Joon, Sung-Hee Park, SangDo Ha, Keun-Sung Kim. Detection of methicillin resistance

Table 1: Methicillin susceptibility of SCV noted isolates in the study

\begin{tabular}{|c|c|c|}
\hline \multirow{2}{*}{$\begin{array}{l}\text { Methicillin suscep- } \\
\text { tibility }\end{array}$} & \multicolumn{2}{|c|}{ S.aureus isolates $(\mathrm{n}=1000)$} \\
\hline & HA $(n=500)$ & $\mathrm{CA}(\mathrm{n}=500)$ \\
\hline Sensitive & 148 & 337 \\
\hline Resistant & 352 & 163 \\
\hline
\end{tabular}

Table 2: Susceptibility pattern of SCV to other antimicrobials $(n=6)$

\begin{tabular}{lllll} 
Isolate & \multicolumn{3}{c}{ Antimicrobial } \\
Gentamicin Linezolid & $\begin{array}{c}\text { Trimetho- } \\
\text { prim-sul- } \\
\text { phamethox- } \\
\text { azole }\end{array}$ & $\begin{array}{c}\text { Cipro- } \\
\text { floxacin }\end{array}$ \\
SCV & $1(16.66 \%)$ & $6(100 \%)$ & $2(33.33 \%)$ & $3(50 \%)$ \\
\hline
\end{tabular}

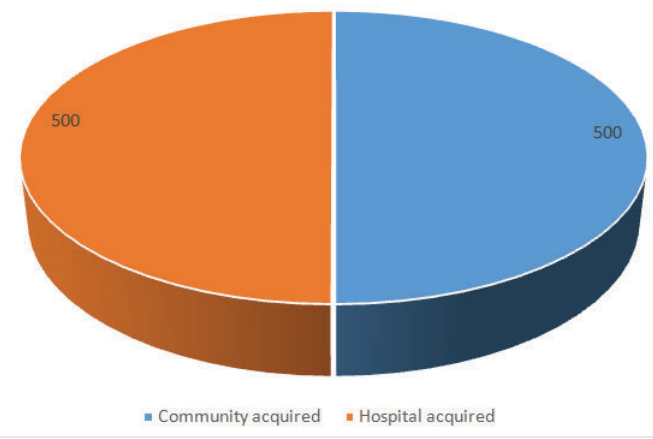

Figure 1: S.aureus isolates included in the study $(n=1000)$. in Staphylococcus aureus isolates using two-step Triplex PCR and conventional methods. J. Microbiol. Biotechnol. 2007; 17(4):673-676.

10. Richard Proctor A., Christof von E, Barbara Kahl C. , Karsten B, Peter N, Mathias H and Georg P.Small colony variants: a pathogenic form of bacteria that facilitates persistent and recurrent infections.Nature Reviews, Microbiology 2006; 4:295-305.

11. Daniel Wolter J., Julia Emerson C. McNamara S, Anne Buccat M. et al Staphylococcusaureus Small-Colony Variants Are Independently Associated With Worse Lung Disease in Children With Cystic Fibrosis. Clinical Infectious Disease 2013; 57(3): 384-391.

12. Looney WJ. Small-colony variants of Staphylococcus aureus. $\mathrm{Br}$ J Biomed Sci. 2000; 57(4):317-322.

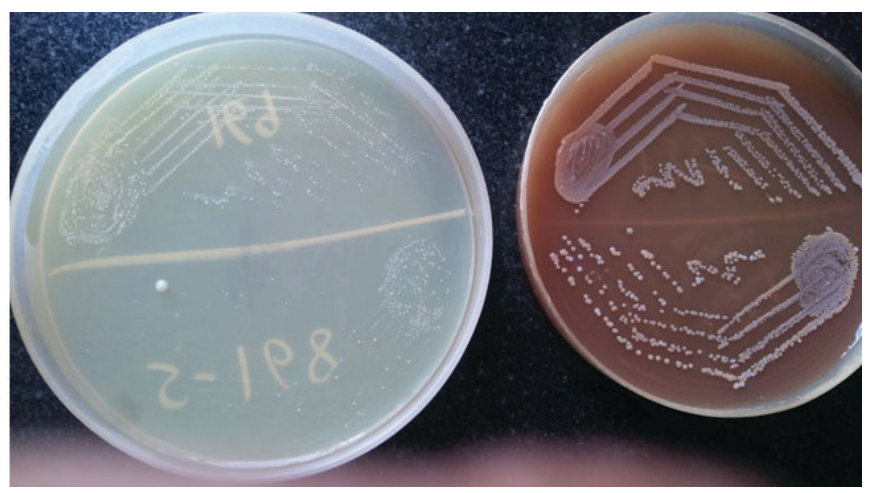

Figure 2: Small colony variants of S.aureus on BHI Agar (with $5 \% \mathrm{NaCl}$ ) and Schaedler's Agar.

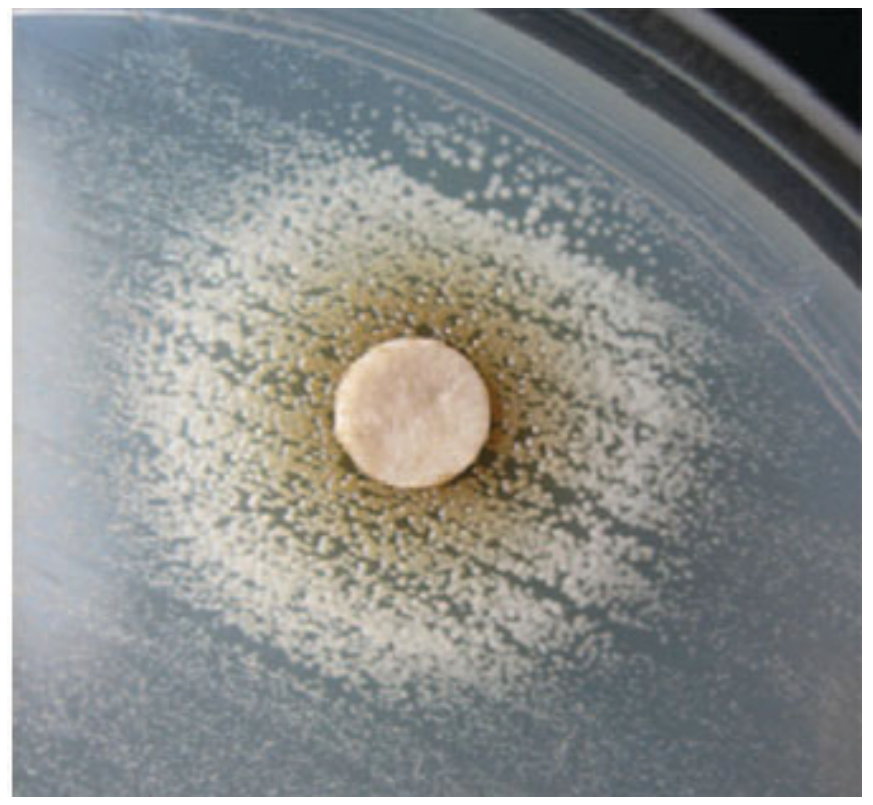

Figure 3: Auxotrophy for Hemin. 


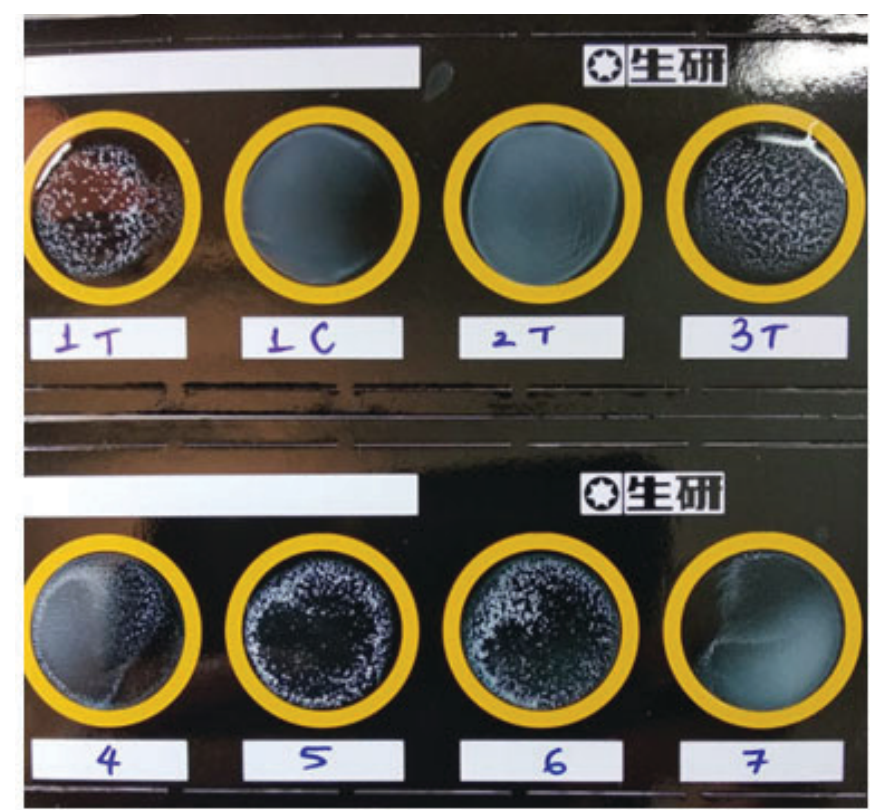

Figure 4: MRSA Screen (Latex Agglutination).

Clumps noted in 1T, 3T, 4, 5 and 6; 1C represents Negative Control

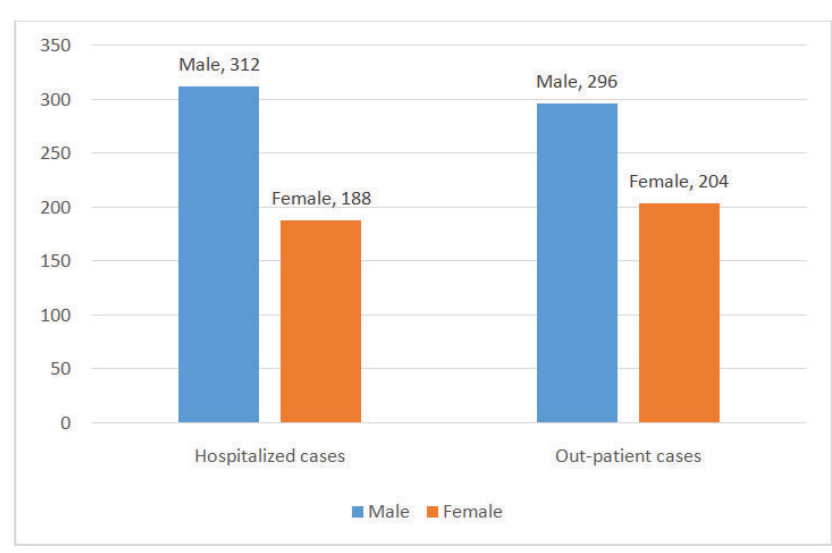

Figure 5: Gender distribution of cases.

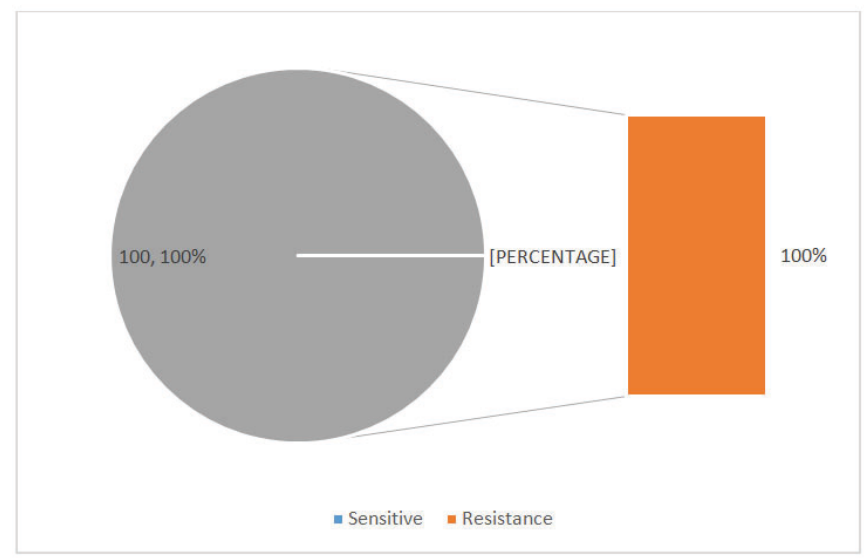

Figure 6: Methicillin susceptibility among SCV $(n=6)$.

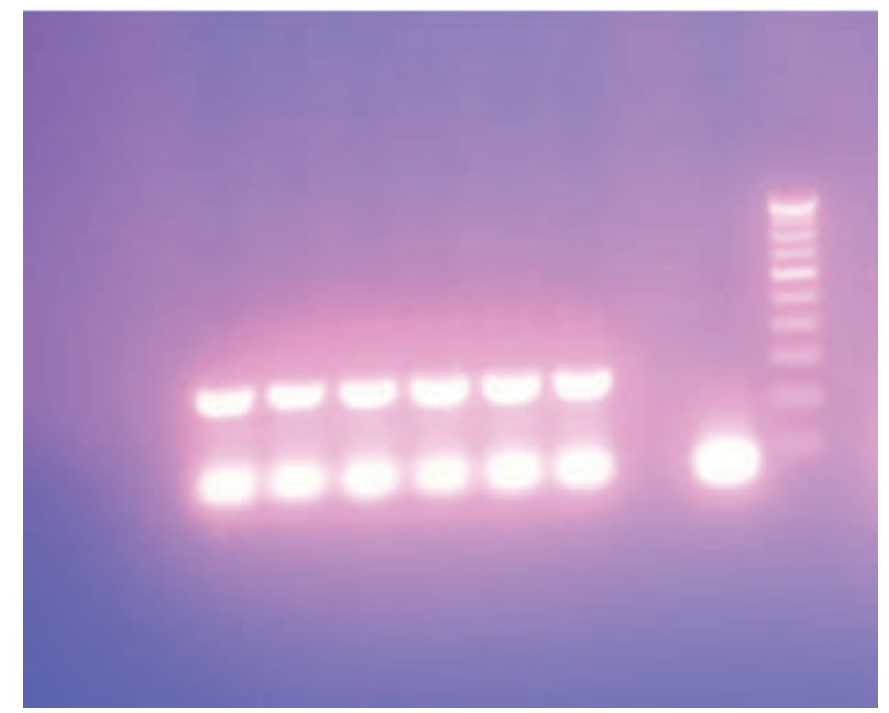

Figure 7: nuc gene detection by conventional PCR.

Lane 1,2,3,4,5,6- nuc positive SCV isolates (270 bp); Lane7,blank; 8-negative control; Lane 9 - Ladder

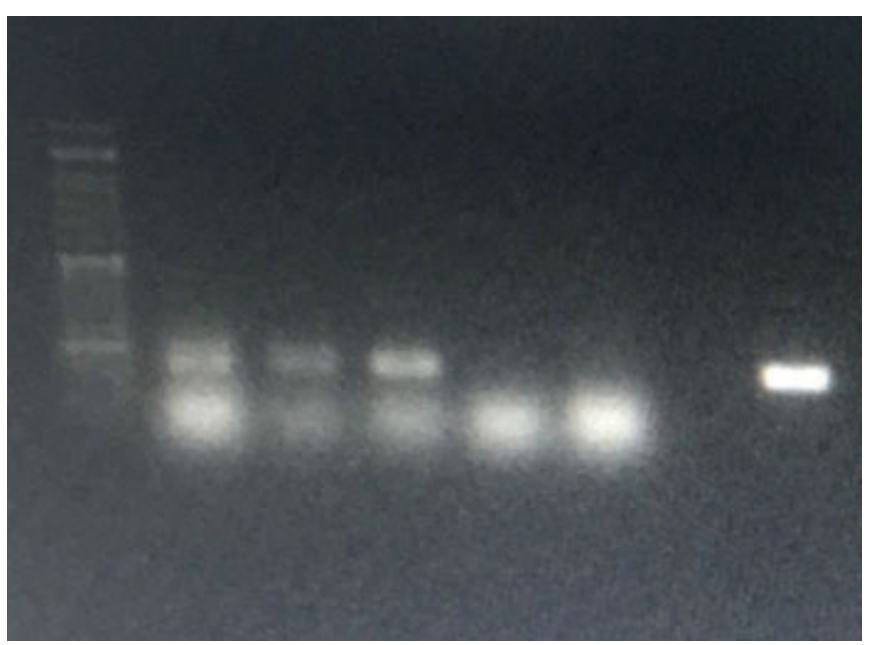

Figure 8: mecA gene detection by conventional PCR.

Lane 1-50bp ladder; Lane 2,3,4 - mecA positive isolates; Lane 5,6-mecA negative isolates; Lane7-negative control; Lane 8 $-m e c A$ positive control (162 bp) 